\title{
Coarsening and Persistence in the Voter Model
}

\author{
E. Ben-Naim ${ }^{1}$, L. Frachebourg ${ }^{2}$, and P. L. Krapivsky ${ }^{3}$ \\ ${ }^{1}$ The James Franck Institute, The University of Chicago, Chicago, IL 60637 \\ ${ }^{2}$ Center for Polymer Studies and Department of Physics, Boston University, Boston, MA 02215 \\ ${ }^{3}$ Courant Institute of Mathematical Sciences, New York University, New York, NY 10012
}

\begin{abstract}
We investigate coarsening and persistence in the voter model by introducing the quantity $P_{n}(t)$, defined as the fraction of voters who changed their opinion $n$ times up to time $t$. We show that $P_{n}(t)$ exhibits scaling behavior that strongly depends on the dimension as well as on the initial opinion concentrations. Exact results are obtained for the average number of opinion changes, $\langle n\rangle$, and the autocorrelation function, $A(t) \equiv \sum(-1)^{n} P_{n} \sim t^{-d / 2}$ in arbitrary dimension $d$. These exact results are complemented by a mean-field theory, heuristic arguments and numerical simulations. For dimensions $d>2$, the system does not coarsen, and the opinion changes follow a nearly Poissonian distribution, in agreement with mean-field theory. For dimensions $d \leq 2$, the distribution is given by a different scaling form, which is characterized by nontrivial scaling exponents. For unequal opinion concentrations, an unusual situation occurs where different scaling functions correspond to the majority and the minority, as well as for even and odd $n$.
\end{abstract}

Typeset using REVTEX 


\section{INTRODUCTION}

The theory of phase separation, or domain coarsening, has undergone a significant development in the last three decades [1]. The most important finding is that well-defined ordered domains arise and grow with time in such a way that the coarsening process exhibits scaling. In other words, at the late stages of the evolution the system is characterized by a single length scale $L(t)$ that gives a typical linear size of the domains. It is well established, at least for systems with a scalar order parameter, that $L(t) \sim t^{n}$ with $n=1 / 2$ for non-conserved dynamics and $n=1 / 3$ for conserved dynamics. For the Ising spin model, Glauber spin-flip dynamics exemplifies the former, while Kawasaki spin-exchange dynamics exemplifies the later.

Several important correlation functions exist. One such function, the autocorrelation or, equivalently, the two-time equal-space correlation function, $A(t)$, is defined by $A(t)=$ $\langle\phi(\mathbf{r}, 0) \phi(\mathbf{r}, t)\rangle$, where $\phi(\mathbf{r}, t)$ is the order parameter. Then, scaling implies: $A(t) \sim L^{-\lambda}(t)$, with an exponent $\lambda[2]$. The general two-point correlation function, $g(r, t)=\langle\phi(\mathbf{0}, 0) \phi(\mathbf{r}, t)\rangle$, can be expressed through $\lambda$, namely $g(r, t)=L^{-\lambda} G(r / L)$. Exact results for $\lambda$ are known in few cases [3] [6], while the bounds $d / 2 \leq \lambda \leq d$ with $d$ the spatial dimension were proposed by Fisher and Huse [2]. For the $O(m)$ vector model in the $m \rightarrow \infty$ limit, $\lambda=d / 2$ [4]. In this study, the value $\lambda=d$ is obtained for the voter model, defined below. This result indicates that both the upper and the lower bounds can be realized.

It should be noted that in most coarsening processes the dynamics does not exhibit a qualitative dependence on the temperature $T$ as long as $T<T_{c}$ [1,7]. At the critical temperature $T=T_{c}$, the dynamics is generally different, and ordered domains usually do not occur. However, the correlation length $\xi(t)$ exists and grows with time as $\xi(t) \sim t^{1 / z}$, where $z$ is the dynamical exponent. The correlation length $\xi(t)$ should be considered as the analog of the domain size $L(t)[5,8]$, and the the exponent $\lambda$ is replaced by $\lambda_{c}$ defined by $A_{c}(t) \sim \xi^{-\lambda_{c}}$. In the voter model, temperature is absent but since the dynamics is noiseless, the voter model dynamics is zero temperature in nature. However, the "critical" temperature 
is also zero. If one introduces noise by allowing environment-independent opinion changes, the system does not coarsen (see, e.g., [9]). Thus, we will actually establish $\lambda_{c}=d$ for the voter model. A general discussion of the conditions under which the equality $\lambda=\lambda_{c}=d$ holds is given by Majumdar and Huse [10].

In this study, we introduce a family of quantities which provides insight into the "history" the the coarsening process. We denote by $P_{n}(t)$ the fraction of voters who changed their opinion exactly $n$ times during the time interval $(0, t)$. The first of these quantities, $P_{0}(t)$, is equal to the fraction of persistent voters, i.e. voters who did not change their opinion up to time $t$. This quantity has been introduced independently for two equivalent onedimensional models, the Glauber-Ising model [11], and the single-species annihilation process [12]. Furthermore, the corresponding generalizations to arbitrary dimensions were discussed in [13] and [14], respectively. Derrida et al. [15] established the exact asymptotic decay of this quantity, $P_{0}(t) \sim t^{-3 / 8}$, as suggested earlier by numerical simulations. Another exact result [16] establishes $P_{0}(t) \sim L^{-\beta}$, with $\beta=0.175075 \ldots$, for the $1 \mathrm{D}$ time-dependent Ginsburg-Landau equation at zero temperature.

For the voter model, several quantities such as the one-time and the two-time correlations are exactly solvable in arbitrary dimensions [9, 18]. These correlation functions allow an exact calculation of the average number of opinion changes $\langle n\rangle$ and other interesting quantities. Hence, the voter model is a natural starting point for investigation of $P_{n}(t)$. Although we do not obtain the full distribution, most of its features are illuminated by combining the above exact results with heuristic arguments and with the mean-field solution. Generally, $P_{n}(t)$ exhibits a scaling behavior. For $d>2$, the scaling function is Poissonian and is peaked at $n=\langle n\rangle$, while for $d=1$ the distribution is maximal near the origin. Additionally, for unequal opinion concentrations different scaling functions for even and odd opinion changes are found. Using random walks techniques, we obtain the even and odd scaling functions in the limit of an infinitesimal minority concentration.

The rest of this paper is organized as follows. In Sec. II, we first solve for $P_{n}(t)$ on a complete graph. We then reexpress some exact relationships for the voter model, in arbitrary 
dimension $d$, in terms of the distribution $P_{n}(t)$. Combining with the mean-field solution, these exact relationships allow us to guess the scaling form of $P_{n}(t)$. This guess suggests a usual scaling form in one dimension, and a mean-field like sharply peaked distribution for $d>1$. These predictions are then compared with numerical data in one, two, and three dimensions. In Sec. III, we describe exact solution of the mean-field equations for the case of initially different concentrations. Then we present exact results for the autocorrelation function in arbitrary dimension $d$, and exact results for the fraction of persistent voters $P_{0}(t)$ in one dimension. We proceed by investigating the extreme case of infinitesimal minority opinion. In this limit, the model is equivalent to a pair of annihilating random walkers who are nearest neighbor at $t=0$. Simplifying further the problem to the case of a random walker near the absorbing boundary we derive a complete analytical solution. Finally, we perform numerical simulations for the case of unequal concentrations and confront the results to exact predictions. Finally, we give a brief summary in Sec. IV.

\section{EQUAL CONCENTRATIONS}

In this section we first define the voter model. We restrict attention to the symmetric case, i.e., equal opinion densities. We start by analyzing the mean-field theory of the model, and then obtain several exact results in arbitrary dimensions. We then present a scaling ansatz and check it using numerical simulations.

\section{A. Mean-Field Theory}

We start by defining the voter model [17]. Consider an arbitrary lattice and assume that each site is occupied by a "voter" who may have one of two opinions, denoted by + and -. Each site keeps its opinion during some time interval, distributed exponentially with a characteristic time $\tau$, set to unity for convenience, and then assumes the opinion of a randomly chosen neighboring site. If a site is surrounded by sites with the same opinion, it does not change its opinion. Hence, such dynamics are zero-temperature in nature. Noise 
can be introduced by allowing a voter to change its opinion independently of its neighbors. However, a voter system with noisy dynamics does not coarsen, and we restrict ourselves to the noiseless voter dynamics. These dynamics are so simple that they naturally arise in a variety of situations, see e.g. [9:[17]. An important link is with the Glauber-Ising model: In one dimension, and only in 1D, the voter dynamics is identical to the Glauber dynamics. This equivalence is not restricted to zero temperature, $1 \mathrm{D}$ noisy voter dynamics is also identical to Glauber dynamics at a positive temperature.

We now consider the voter model dynamics on a mean-field level, by simply treating all sites as neighbors. Such a theory is exact on a complete graph. Moreover, it is expected to hold in sufficiently large spatial dimensions. We first consider the symmetric case were the opinions concentrations, $c_{+}$and $c_{-}$, are equal, and the interesting case of unequal concentrations will be discussed later. The fraction of sites which have changed their opinions $n$ times up to time $t$, evolves according to

$$
\frac{d P_{n}}{d t}=P_{n-1}-P_{n}
$$

with $P_{-1} \equiv 0$ to ensure $d P_{0} / d t=-P_{0}$. The distribution clearly satisfies the normalization condition, $\sum_{n} P_{n}=1$, and one can verify that Eq. (1) conserves this sum. Solving Eq. (11) subject to the initial condition $P_{n}(0)=\delta_{n 0}$, one finds that the opinion change distribution function is Poissonian

$$
P_{n}(t)=\frac{t^{n}}{n !} e^{-t}
$$

In particular, the fraction of persistent voters, i.e. voters who did not change their opinion up to time $t$, decreases exponentially, $P_{0}(t)=e^{-t}$. The probability that a voter has its initial opinion at time $t$ is thus $P_{\text {even }}=\sum_{n} P_{2 n}=\left(1+e^{-2 t}\right) / 2$. Asymptotically, this probability exponentially approaches the value $1 / 2$, and therefore voters quickly "forget" their initial opinion.

The distribution is peaked around the average $\langle n\rangle=t$, and the width of the distribution, $\sigma$, is given by $\sigma^{2}=\left\langle n^{2}\right\rangle-\langle n\rangle=t$. In the limits, $t \rightarrow \infty, n \rightarrow \infty$, and $(n-t) / \sqrt{t}$ finite, $P_{n}(t)$ approaches a scaling form 


$$
P_{n}(t)=\frac{1}{\sigma} \Phi_{\infty}\left(\frac{n-\langle n\rangle}{\sigma}\right)
$$

where the scaling distribution is Gaussian $\Phi_{\infty}(x)=(2 \pi)^{-1 / 2} \exp \left(-x^{2} / 2\right)$. This infinitedimension scaling solution will be compared below to simulation results in three dimensions. To summarize, the quantity $P_{n}(t)$ incorporates many statistical properties of the system such as the probability of maintaining the original opinion, the probability of having the original opinion, and the average number of opinion changes.

\section{B. Exact Results}

We now review several relevant known exact results for the voter model in arbitrary dimension $d$ and reexpress them in terms of $P_{n}(t)$. Both the one- and two-body equal-time correlation functions [9, 18], are exactly solvable on arbitrary lattice in arbitrary dimension. It proves useful to formulate the voter model on the language of Ising spins, i.e., a + opinion is identified with +1 spin and a - opinion with -1 spin. The state of the lattice is described by $S \equiv\left[S_{\mathbf{k}}\right]$, the spin-flip rate $W_{\mathbf{k}}(S) \equiv W\left(S_{\mathbf{k}} \rightarrow-S_{\mathbf{k}}\right)$ reads

$$
W_{\mathbf{k}}(S)=1-\frac{1}{z_{d}} S_{\mathbf{k}} \sum_{\mathbf{e}_{i}} S_{\mathbf{k}+\mathbf{e}_{i}}
$$

with $z_{d}$ the coordination number. Here the sum in the right-hand side runs over all $z_{d}$ nearest neighbors. It is convenient to rescale the time variable, $t \rightarrow t z_{d} / 4$. The probability distribution $P(S, t)$ satisfies the master equation

$$
\frac{d}{d t} P(S, t)=\sum_{\mathbf{k}}\left[W_{\mathbf{k}}\left(S^{\mathbf{k}}\right) P\left(S^{\mathbf{k}}, t\right)-W_{\mathbf{k}}(S) P(S, t)\right],
$$

where the state $S^{\mathbf{k}}$ differs from $S$ only at the site $\mathbf{k}$. One can then derive a set of differential equations for the spin correlation functions $\left\langle S_{\mathbf{k}} \ldots S_{\mathbf{l}}\right\rangle \equiv \sum_{S} S_{\mathbf{k}} \ldots S_{\mathbf{l}} P(S, t)$. The single- and two-body correlation functions satisfy discrete Laplace equations [9],

$$
\begin{aligned}
2 \frac{d}{d t}\left\langle S_{\mathbf{k}}\right\rangle & =-z_{d}\left\langle S_{\mathbf{k}}\right\rangle+\sum_{\mathbf{e}_{i}}\left\langle S_{\mathbf{k}+\mathbf{e}_{i}}\right\rangle \\
2 \frac{d}{d t}\left\langle S_{\mathbf{k}} S_{\mathbf{l}}\right\rangle & =-2 z_{d}\left\langle S_{\mathbf{k}} S_{\mathbf{l}}\right\rangle+\sum_{\mathbf{e}_{i}}\left\langle S_{\mathbf{k}+\mathbf{e}_{i}} S_{\mathbf{l}}\right\rangle+\sum_{\mathbf{e}_{i}}\left\langle S_{\mathbf{k}} S_{\mathbf{l}+\mathbf{e}_{i}}\right\rangle .
\end{aligned}
$$


On a simple (hyper)cubic lattice where $z_{d}=2 d$, the general solution for the average opinion is given by

$$
\left\langle S_{\mathbf{k}}\right\rangle=e^{-t d} \sum_{\mathbf{l}}\left\langle S_{\mathbf{l}}(0)\right\rangle I_{\mathbf{k}-\mathbf{l}}(t)
$$

where $I_{\mathbf{k}}(x)$ is the multi-index function, $I_{\mathbf{k}}(x)=\prod_{1 \leq j \leq d} I_{k_{j}}(x)$, and $I_{n}$ is the modified Bessel function. The autocorrelation $A(t)=\left\langle S_{\mathbf{0}}(0) S_{\mathbf{0}}(t)\right\rangle$ is of particular interest since it is related to the opinion change distribution via the alternating sum $A(t)=\sum(-1)^{n} P_{n}(t)$. The autocorrelation is found from Eq. (6), $A(t)=e^{-t d} \sum_{\mathbf{l}}\left\langle S_{\mathbf{0}}(0) S_{\mathbf{l}}(0)\right\rangle I_{\mathbf{l}}(t)$. In the simplest case of completely uncorrelated initial opinions, with equal densities of the opposite opinions, $\left\langle S_{\mathbf{0}}(0) S_{\mathbf{l}}(0)\right\rangle=\delta_{\mathbf{l 0}}$, one finds

$$
A(t)=\sum_{n}(-1)^{n} P_{n}(t)=\left[e^{-t} I_{0}(t)\right]^{d}
$$

and thus asymptotically, $A(t) \simeq(2 \pi t)^{-d / 2}$. The diffusive nature of the problem (see e.g. Eq. (6) ) suggests that the correlation length is given by the diffusion scale, $\xi(t) \sim \sqrt{t}$. Therefore, the autocorrelation function scales as $\xi^{-d}$ for arbitrary $d$, thus implying that the exponent $\lambda_{c}$ is well defined in all dimensions, and equal to $\lambda_{c}=d$ as claimed previously.

The average number of opinion changes, $\langle n\rangle=\sum_{n} n P_{n}$, is simply related to the concentration of "active bonds" (neighbors with different opinions) $c_{+-} \equiv\left(1-\left\langle S_{\mathbf{l}} S_{\mathbf{l}+\mathbf{e}}\right\rangle\right) / 2$ : $d\langle n\rangle / d t=c_{+-}$. Evaluation of the active bonds density gives the following leading asymptotic behavior 18

$$
c_{+-}(t) \sim \begin{cases}t^{-1+d / 2}, & d<2 \\ 1 / \ln t, & d=2 \\ \text { const, } & d>2\end{cases}
$$

Thus, when $d \leq 2$, the density of active bonds vanishes for sufficiently long-time, i.e. coarsening takes place in low dimensions. In contrast, for $d>2$, single-opinion domains do not arise. This is not very surprising since at the critical point well-ordered domains should not necessarily form. Following the above discussion, the average number of opinion changes in the limit $t \rightarrow \infty$ is obtained by integrating $c_{+-}$, 


$$
\langle n\rangle \sim \begin{cases}t^{d / 2}, & d<2 \\ t / \ln t, & d=2 \\ t, & d>2 .\end{cases}
$$

The above results agree with the mean-field results when $d>2$. We therefore expect that for $d>2$, the distribution function $P_{n}(t)$ approaches the Poissonian distribution of Eq. (3). Similarly, the fraction of persistent voters $P_{0}(t)$ should decay exponentially in time as well. Interestingly, the exact result for the autocorrelation function indicates a subtle failure of the mean-field approach concerning the probability that a voter has its initial opinion $P_{\text {even }}(t)=\sum P_{2 n}(t)=(1+A(t)) / 2$. From Eq. (8), one finds that asymptotically $P_{\text {even }}(t)-1 / 2 \sim t^{-d / 2}$, while the mean-field approach gives $P_{\text {even }}(t)-1 / 2 \sim e^{-2 t}$. Hence voters have a stronger than exponential memory, even for $d>2$. Despite this erroneous prediction, the mean-field theory is successful in predicting most features of the opinion change distribution function for $d>2$.

\section{Scaling Arguments}

We were unable to find the exact $P_{n}(t)$ distribution for $d \leq 2$, or higher moments such as $\left\langle n^{2}\right\rangle$. However, combining the above results with scaling arguments proves useful. In one dimension, the average number of changes scales as $\langle n\rangle \sim \sqrt{t}$. We assume that this scale characterizes the distribution, or in other words, $\sigma \sim\langle n\rangle \sim \sqrt{t}$. Thus, we arrive at the scaling form

$$
P_{n}(t)=\frac{1}{\sqrt{t}} \Phi_{1}\left(\frac{n}{\sqrt{t}}\right) .
$$

In general we will use the notation $\Phi_{d}$ for the $d$-dimensional scaling function. The nontrivial decay of the persistent voter density, $P_{0} \sim t^{-3 / 8}$ [15], implies nontrivial divergence of the scaling form $\Phi_{1}(z) \sim z^{-1 / 4}$, in the small argument limit, $z \rightarrow 0$. The tail of the distribution corresponds to a large number of opinion changes by a specific voter and can be estimated by an intuitive argument. Such a voter must reside at the boundary between two single opinion 
regimes, and must change its mind constantly. The probability that such a voter changes its mind $t$ times (one time per unit time), can be estimated by $P_{t}(t)=\exp (-$ const $\times t)$. It is also natural to assume that the scaling function rapidly decays for large $z, \Phi_{1}(z) \sim$ $\exp \left(-\right.$ const $\left.\times z^{\alpha}\right)$. Combining this form with Eq. (11) gives $P_{t}(t) \sim \exp \left(-\right.$ const $\left.\times t^{\alpha / 2}\right)$ and consequently, $\alpha=2$. To summarize, the limiting behavior of the one-dimensional scaling function are

$$
\Phi_{1}(z) \sim \begin{cases}z^{-1 / 4} & z \ll 1 \\ \exp \left(-\mathrm{const} \times z^{2}\right) & z \gg 1 .\end{cases}
$$

This scaling behavior is different in nature than the scaling behavior for dimensions $d>2$. While for $d \geq 2$, a well-defined peak in the distribution function occurs near the average, the one dimensional distribution is a decreasing function of $n$. Moreover, the Gaussian function $\Phi_{\infty}(z)=(2 \pi)^{-1 / 2} \exp \left(-z^{2}\right)$ is symmetric around its average, while no such symmetry occurs

for $d=1$, as the distribution is peaked near $n=0$. Despite these differences, the tail of the distribution of Eq. (12) agrees with the mean-field distribution of Eq. (3). In fact, the above heuristic argument for the large- $n$ behavior is valid in arbitrary dimensions.

\section{Simulation Results}

We implement the voter model using a simple Monte-Carlo simulation. A simple (hyper)cubic lattice is chosen with a linear size $L$, and periodic boundary conditions are imposed. A simulation step consists of choosing randomly an active bond (i.e., a bond between neighbors with different opinions) and changing the opinion of one of the two voters. After each such step, time is incremented by the inverse number of active bonds and the active bond list is updated. This simulation procedure is efficient for spatial dimensions $d \leq 2$ since the system coarsens and the number of active bonds decreases as the simulation proceeds. The results below correspond to one realization on a lattice of linear dimension $L=10^{7}, 10^{3}$, and $2 \times 10^{2}$ in $1 \mathrm{D}, 2 \mathrm{D}$, and $3 \mathrm{D}$, respectively.

In one dimension, the numerical results confirm the scaling ansatz of Eq. (11), as shown in Fig. 1. Interestingly, the maximum of the distribution occurs at $n=1$, and the distribution 
decays monotonously for $n>1$. The postulated limiting behaviors of the scaling distribution are also confirmed. To test the validity of the mean-field theory, we performed numerical simulations in three dimensions. The resulting $P_{n}(t)$ distribution agrees with the Poissonian distribution of Eq. (2), and furthermore the fraction of persistent voters decays exponentially. These results indicate that the above mentioned discrepancy regarding the autocorrelation function is not crucial in understanding the opinion change distribution function.

The marginal two-dimensional case is especially interesting. While it is expected that the distribution will be roughly Poissonian, some deviation from the mean-field predictions are expected. We find numerically that the distribution obeys the scaling form of Eq. (3), and exhibits a well defined peak in the vicinity of $\langle n\rangle \sim t / \ln t$. However, in 2D, the system still coarsens, and the distribution exhibits some low- dimensional features. The distribution is not a symmetric function of the variable $n-\langle n\rangle$ (Fig. 2). Additionally, an intriguing behavior for the fraction of persistent voters is found numerically (Fig. 3),

$$
P_{0}(t) \sim \exp \left(- \text { const } \times \ln ^{2} t\right)
$$

Thus the fraction of persistent voters decays faster than the 1D power-law behavior, and slower than the mean-field exponential behavior and a naive logarithmic correction to the mean-field behavior does not hold. The width of the distribution $\sigma$ is not given by a logarithmic correction to the mean-field (where $\sigma \sim t^{1 / 2}$ ), and our best fit gives $\sigma \sim t / \ln ^{\alpha} t$ with $\alpha>1$. The $2 \mathrm{D}$ case is difficult from a numerical point of view since logarithmic corrections occur, and a large temporal range is required to distinguish such slowly varying corrections from algebraic behavior with small exponents [18].

\section{UNEQUAL CONCENTRATIONS}

Our previous exposition has assumed that the initial concentrations of dissimilar species are equal, $c_{+}=c_{-}=1 / 2$. The case of unequal concentrations, $c_{+} \neq c_{-}$, is interesting as well. The reason is that the voter model dynamics has a remarkable feature: Although locally the 
opinion does change (the dynamics is non-conserved in nature), globally both opinions are conserved. On the language of the Ising model it can be said that at zero temperature the magnetization remains constant. This hidden integral leads to several peculiarities which will be illuminated first on a mean-field level and then for a special case in one dimension.

\section{A. Mean-Field Theory}

To write the general mean-field theory, it is necessary to distinguish between voters according to their initial opinions. Hence, we introduce $P_{n}^{+}(t)\left(P_{n}^{-}(t)\right)$, the fraction of voters with the $+(-)$ initial opinion that have changed their opinion $n$ times up to time $t$. If all sites are neighbors, these distributions evolve according to

$$
\begin{aligned}
\frac{d P_{2 n}^{+}}{d t} & =2\left(c_{+} P_{2 n-1}^{+}-c_{-} P_{2 n}^{+}\right), \\
\frac{d P_{2 n+1}^{+}}{d t} & =2\left(c_{-} P_{2 n}^{+}-c_{+} P_{2 n+1}^{+}\right), \\
\frac{d P_{2 n}^{-}}{d t} & =2\left(c_{-} P_{2 n-1}^{-}-c_{+} P_{2 n}^{-}\right), \\
\frac{d P_{2 n+1}^{-}}{d t} & =2\left(c_{+} P_{2 n}^{-}-c_{-} P_{2 n+1}^{-}\right),
\end{aligned}
$$

with $P_{-1}^{ \pm} \equiv 0$. The initial conditions are $P_{n}^{ \pm}(t=0)=c_{ \pm} \delta_{n 0}$. These rate equations reduce to Eq. (11) for the symmetric case $c_{+}=c_{-}=1 / 2$.

It is again useful to consider the fraction of voters that have (do not have) their initial opinion, $P_{\text {even }}^{ \pm}=\sum_{n} P_{2 n}^{ \pm}\left(P_{\text {odd }}^{ \pm}=\sum_{n} P_{2 n+1}^{ \pm}\right)$. Summation of Eqs. (14) gives

$$
\frac{d P_{\mathrm{even}}^{ \pm}}{d t}=-\frac{d P_{\mathrm{odd}}^{ \pm}}{d t}=2 c_{ \pm}^{2}-2 P_{\mathrm{even}}^{ \pm}
$$

One can find that the global opinion concentrations $c_{ \pm}=P_{\text {even }}^{ \pm}+P_{\text {odd }}^{\mp}$ are conserved and that $P_{\text {odd }}^{+}=P_{\text {odd }}^{-}$. Solving these last rate equations subject to the proper initial conditions gives

$$
\begin{gathered}
P_{\text {even }}^{ \pm}=c_{ \pm}\left(c_{ \pm}+c_{\mp} e^{-2 t}\right), \\
P_{\text {odd }}^{ \pm}=c_{+} c_{-}\left(1-e^{-2 t}\right) .
\end{gathered}
$$


The autocorrelation function is then given by

$$
\begin{array}{r}
A(t)=P_{\text {even }}^{+}+P_{\text {even }}^{-}-P_{\text {odd }}^{+}-P_{\text {odd }}^{-} \\
=\left(c_{+}-c_{-}\right)^{2}+4 c_{+} c_{-} e^{-2 t} .
\end{array}
$$

A voter quickly forgets its initial opinion even if statistically it is more likely to have its initial opinion since $c_{+}^{2}+c_{-}^{2} \geq 2 c_{+} c_{-}$.

The fraction of persistent voters is found by solving $d P_{0}^{ \pm} / d t=-2 c_{\mp} P_{0}^{ \pm}$and it is found that

$$
P_{0}^{ \pm}=c_{ \pm} e^{-2 c_{\mp} t}
$$

Thus, the fraction of persistent voters decays exponentially as well. The decay constant is simply given by the density of opposite opinion. This result indicates that even in the case of a small concentration of one opinion, the fraction of persistent majority voters decays exponentially with time.

To solve Eqs. (14) we introduce the generating functions

$$
\begin{aligned}
& F_{\text {even }}^{ \pm}(t, w)=\sum_{n=0}^{\infty} P_{2 n}^{ \pm}(t) w^{2 n} \\
& F_{\text {odd }}^{ \pm}(t, w)=\sum_{n=0}^{\infty} P_{2 n+1}^{ \pm}(t) w^{2 n+1}
\end{aligned}
$$

This reduces the infinite set of rate equations to four equations

$$
\begin{aligned}
& \frac{d F_{\text {even }}^{ \pm}}{d t}=2\left(c_{ \pm} w F_{\text {odd }}^{ \pm}-c_{\mp} F_{\text {even }}^{ \pm}\right) \\
& \frac{d F_{\text {odd }}^{ \pm}}{d t}=2\left(c_{\mp} w F_{\text {even }}^{ \pm}-c_{ \pm} F_{\text {odd }}^{ \pm}\right) .
\end{aligned}
$$

Expressing $F_{\text {odd }}^{ \pm}$via $F_{\text {even }}^{ \pm}$, we reduce the system of first-order differential equations (20) to a pair of second-order equations for $F_{\mathrm{even}}^{+}(t, w)$ and $F_{\text {even }}^{-}(t, w)$. Solving these equations subject to the proper boundary conditions yields

$$
F_{\text {even }}^{ \pm}(t, w)=c_{ \pm} e^{-t}\left(\cosh (t \Delta) \pm\left(c_{+}-c_{-}\right) \frac{\sinh (t \Delta)}{\Delta}\right)
$$


where a shorthand notation, $\Delta=\sqrt{1-4 c_{+} c_{-}\left(1-w^{2}\right)}$, has been used. In principle, one then finds $P_{n}^{+}(t)$ and $P_{n}^{-}(t)$ by expanding the generating functions. This leads to rather cumbersome results. However, the most interesting scaling results correspond to the limit $t \rightarrow \infty, 1-w \rightarrow+0$ with $(1-w) t$ kept finite. In this scaling limit, $1-\Delta \rightarrow 4 c_{+} c_{-}(1-w) t$. Substituting this into Eq. (21) we find $F_{\text {even }}^{ \pm} \simeq c_{+}^{2} \exp \left(-4 c_{+} c_{-}(1-w) t\right)$. Then we find $F_{\text {odd }}^{ \pm}$, note that in the scaling limit the generating functions become the Laplace transforms of $P_{n}^{+}(t)$ and $P_{n}^{-}(t)$, and perform the inverse transformation. Finally, we arrive at the following scaling results

$$
\begin{aligned}
\frac{P_{2 n}^{+}}{c_{+}^{2}} \simeq \frac{P_{2 n}^{-}}{c_{-}^{2}} \simeq & \frac{P_{2 n+1}^{+}}{c_{+} c_{-}} \simeq \frac{P_{2 n+1}^{+}}{c_{+} c_{-}} \simeq \\
& \frac{1}{\sqrt{2 \pi c_{+} c_{-} t}} \exp \left[-\frac{\left(n-2 c_{+} c_{-} t\right)^{2}}{2 c_{+} c_{-} t}\right]
\end{aligned}
$$

In particular, we see that for $c_{+} \neq c_{-}$the distribution function for the even number of changes, $P_{2 n}=P_{2 n}^{+}+P_{2 n}^{-}$, is larger than the distribution function for the odd number of changes, $P_{2 n+1}=P_{2 n+1}^{+}+P_{2 n+1}^{-}$. Eq. (22) suggests that it is possible to avoid these "evenodd oscillations", by making a transformation to a modified opinion change distribution $\tilde{P}_{n} \equiv P_{n}+P_{n+1}$. We also note that the scaling distribution in the right hand side of Eq. (22) is identical with the infinite-dimension scaling function, previously obtained for the symmetric case.

\section{B. Exact Results}

Although the above results were obtained using mean-field considerations, similar behavior characterizes the exact solution. By generalizing the solution of Eq. (8), the autocorrelation function is found

$$
A(t)=\sum(-1)^{n} P_{n}=\left(c_{+}-c_{-}\right)^{2}+4 c_{+} c_{-}\left[I_{0}(t) e^{-t}\right]^{d}
$$

The limiting value of the autocorrelation function, $\left(c_{+}-c_{-}\right)^{2}$, is identical with the mean-field theory Eq. (17). Again the conclusion remains the same, at the late stages of the process a 
single voter opinion cannot be used to determine its initial opinion. Similar to the symmetric case, the autocorrelation function decays algebraically rather than exponentially with time. Since $P_{\text {even }}=(1+A(t)) / 2 \geq P_{\text {odd }}=(1-A(t)) / 2$, we also learn that a voter is more likely to have its initial opinion.

Mean-field theory suggests that the fraction of persistent voters decays faster for the minority. It is interesting to investigate the same for the one-dimensional situation. It is instructive to start with the special case of $c_{+}=1 / 3$ and $c_{-}=2 / 3$. Let us formally split the - opinion into two equivalent sub-opinions. Hence, there are three equiprobable opinions, one + opinion and two - sub-opinions. We now identify this system as the zero-temperature three states Potts model, or as a voter model with three opinions. The dynamics is unchanged, a voter chooses a nearest neighbor randomly, and assumes its opinion. Eventually, we will not distinguish between the - sub-opinions. For the kinetic $q$-state Potts model with $T=0$, the fraction of persistent spins decays according to $P_{0}(t) \sim t^{-\beta(q)}$, with $\beta(q)=2 \pi^{-2}\left[\cos ^{-1}\left(\sqrt{2} q^{-1}-1 / \sqrt{2}\right)\right]^{2}-1 / 8$, see 15. Indeed, for the symmetric voter model, $q=2$ and $\beta(2)=3 / 8$. The concentration of persistent minority species, $P_{0}^{+}(t)$, is equal to the fraction of persistent spins in the $q$-state Potts model with $q=3$. Using the notation $P_{0}^{ \pm}(t) \sim t^{-\beta_{ \pm}}$, one has $\beta_{+}=\beta(3) \cong 0.5379$. Of course, $\beta_{-} \neq \beta_{+}$, since changes between - sub-opinions should not be counted. The exponent $\beta_{-}$can be found by allowing a non-integer number of opinions, $q=1 / c_{-}=3 / 2$. This formula is found by an analytical continuation to arbitrary $q$ of the relation $c=1 / q$, which clearly holds in the equal-concentration case with an integer $q$. Therefore, $q_{ \pm}=1 / c_{ \pm}$. For the above example, $c_{-}=2 / 3, q_{-}=3 / 2$ implies $\beta_{-} \cong 0.2349$. In general, the concentration of persistent voters decays algebraically

$$
P_{0}^{ \pm} \sim t^{-\beta_{ \pm}} \quad \text { with } \quad \beta_{ \pm}=\frac{2}{\pi^{2}}\left[\cos ^{-1}\left(\sqrt{2} c_{ \pm}-1 / \sqrt{2}\right)\right]^{2}-\frac{1}{8}
$$

Following Eq. (11), $P_{n}(t)$ can be written in terms of a simple scaling function in one dimension. The $z \rightarrow 0$ behavior reflects the anomalously large number of persistent voters found in the system at long times. On the other hand, Eq. (24) implies a difference in nature 
of the scaling functions for sites of initial + and - opinion, $P_{n}^{ \pm}(t)=\Phi_{1}^{ \pm}(n / \sqrt{t}) /\left(c_{ \pm} \sqrt{t}\right)$. In the limit of large $z=n / \sqrt{t}$, the tail is dominated by Gaussian fluctuations, while in the limit $z \rightarrow 0$, the anomalous decay of Eq. (24) determines the behavior. Combining these two limits, we have

$$
\Phi_{1}^{ \pm}(z) \sim \begin{cases}z^{2 \beta\left(c_{ \pm}\right)-1} & z \ll 1 \\ \exp \left(-\mathrm{const} \times z^{2}\right) & z \gg 1\end{cases}
$$

In the limit of a vanishing minority opinion concentration, $c_{+} \rightarrow 0$, one has $\beta_{+} \rightarrow 1$, and $\beta_{-} \cong 2 c_{+} / \pi \rightarrow 0$

Both the mean-field results and our numerical simulations, to be described in the following, suggest that distribution of even number of changes dominates over its odd counterpart. We expect that the above suggested scaling form holds for the even distribution, or equivalently, for the modified distribution $P_{n}+P_{n+1}$. To summarize, the exact form of the fraction of persistent voters combined with scaling considerations suggest that different scaling functions correspond to the minority and the majority opinions.

\section{Infinitesimal concentrations}

For better understanding of the asymmetric case, it is useful to consider the case of an infinitesimal concentration of one opinion, $c_{+} \rightarrow 0$. We naturally restrict ourself to the situation where a single + voter is placed in a sea of - opinion. Identifying an interface between + and - domains with a random walker, an equivalence to two annihilating random walkers who are nearest neighbors at $t=0$, is established. The distribution $P_{n}(t)$ is thus equal to the fraction of sites visited $n$ times by the two walkers. We further simplify the problem by considering the fraction of sites visited by a single random walk with a trap as one of its nearest neighbors. Although the two problems are not identical, we expect that the results are similar in nature and differ only by numerical prefactors. The reason is that the distance between the two random walks itself performs a random walk with in the vicinity of a trap. 
In the limit of a vanishing opinion concentration, $c_{+} \rightarrow 0$, the opinion change density $P_{n}(t)$ is equal to zero. However, if we divide $P_{n}^{-}(t)$ by the density of the interfaces, $c_{+} c_{-}$, and then go to the limit $c_{+} \rightarrow 0$, we obtain a nontrivial distribution, $\lim _{c_{+} \rightarrow 0} P_{n}^{-}(t) / c_{+} c_{-}$. This distribution gives the total number of links crossed $n$ times by the walker; we will denote it by $P_{n}(t)$.

As said previously, for the symmetric initial conditions, $c_{+}=c_{-}=1 / 2$, the scaling behavior of the form $P_{n}(t)=t^{-1 / 2} \Phi_{1}(n / \sqrt{t})$ is expected. However, for asymmetric initial conditions, two different scaling forms, even and odd, should appear. In the present extreme case, we expect $P_{2 n}(t)=t^{-1 / 2} \Phi_{\text {even }}(n / \sqrt{t})$ and $P_{2 n+1}(t)=t^{-1 / 2} \Phi_{\text {odd }}(n / \sqrt{t})$. We learn from Eq.(25) that $\Phi_{\text {even }} \equiv \Phi_{1}^{-} \sim z^{-1}$ near the origin. Hence, the distribution function approaches a time independent form: $\lim _{t \rightarrow \infty} P_{n}(t) \sim n^{-1}$.

These results can be confirmed by considering the analogy to a single random walk near a trap. As the walker will ultimately come to the origin with probability one, every link $(k-1, k), k \geq 2$ will be crossed an even number of time and so the ultimate distribution $P_{2 n+1}(\infty)=0$ for $n \geq 1$ (and $P_{1}(\infty)=1$ since the link $(0,1)$ is crossed with ultimate probability one by the walker). So, in the extreme case we are considering, the even-odd oscillations are obvious and pronounced: The asymptotic even values are positive while the odd values are zero.

In order to compute $P_{n}(\infty)$ for $n$ even, we consider the link $(k-1, k)$. The probability that the walker starting at $x=1$ reaches for the first time $x=k$, thus crossing the link $(k-1, k)$, is given by $p(k)=1 / k$ [20]. Then the ultimate probability that the walker will go from site $x=k$ to site $x=k-1$, crossing the link $(k-1, k)$ a second time, is one. The probability that the walker starting at $x=k-1$ will arrive at $x=0$ before crossing the link $(k-1, k)$ again, is given by $1 / k$. Therefore, $k^{-2}$ is the contribution of the link $(k-1, k)$ into $P_{2}(\infty)$, the average number of links crossed twice by the walker. Thus, we have

$$
P_{2}(\infty)=\sum_{k=2}^{\infty} \frac{1}{k^{2}}=\zeta(2)-1=\frac{\pi^{2}}{6}-1
$$

After having crossed the link $(k-1, k)$ twice, the walker could cross this link again before 
reaching the adsorbing barrier at $x=0$. Any such crossing from the left happens with probability $1-1 / k$, while the next crossing from the right happens with probability one. Thus, we arrive at the remarkably simple formula expressing $P_{n}(\infty)$ through the zeta function

$$
\begin{aligned}
P_{2 n+2}(\infty) & =\sum_{k=2}^{\infty}\left(1-\frac{1}{k}\right)^{n} \frac{1}{k^{2}} \\
& =\sum_{m=0}^{n}\left(\begin{array}{c}
n \\
m
\end{array}\right)(-1)^{m}(\zeta(m+2)-1)
\end{aligned}
$$

For large $n$, the sum can be approximated by the integral

$$
P_{2 n}(\infty) \simeq \int_{0}^{1 / 2}(1-\xi)^{n-1} d \xi=\frac{1-2^{-n}}{n} \simeq \frac{1}{n}
$$

which confirms the above prediction.

To determine the scaling functions $\Phi_{\text {even }}(z)$ and $\Phi_{\text {odd }}(z)$, it proves useful to consider $P_{n}(x, t)$, the probability that the walker passes $n$ times through $x$ during the time interval $(0, t)$. The $P_{n}(t)$ is then given by

$$
P_{n}(t)=\sum_{x=2}^{\infty} P_{n}(x, t) \simeq \int_{2}^{\infty} d x P_{n}(x, t) .
$$

In this equation and in the following we will treat $x$ as a continuous variable; in the long-time limit, this should be asymptotically correct.

We then write for $P_{n}(x, t)$ :

$$
\begin{aligned}
P_{2 n}(x, t) & =\int_{0}^{t} d t_{1} p_{1}\left(x, t_{1}\right) \int_{0}^{t-t_{1}} d t_{2} p_{2}\left(t_{2}\right) \int_{0}^{t-t_{1}-t_{2}} d t_{3} p_{3}\left(x, t_{3}\right) \\
& \cdots \int_{0}^{t-\sum_{i \leq 2 n-1} t_{i}} d t_{2 n} p_{2}\left(t_{2 n}\right) p_{4}\left(x, t-\sum_{i=1}^{2 n} t_{i}\right)
\end{aligned}
$$

and

$$
\begin{gathered}
P_{2 n+1}(x, t)=\int_{0}^{t} d t_{1} p_{1}\left(x, t_{1}\right) \int_{0}^{t-t_{1}} d t_{2} p_{2}\left(t_{2}\right) \int_{0}^{t-t_{1}-t_{2}} d t_{3} p_{3}\left(x, t_{3}\right) \\
\ldots \int_{0}^{t-\sum_{i \leq 2 n} t_{i}} d t_{2 n+1} p_{3}\left(x, t_{2 n}\right) p_{5}\left(t-\sum_{i=1}^{2 n+1} t_{i}\right) .
\end{gathered}
$$

We consider a walker starting at $y_{0}=1 ; p_{1}(x, t)$ is the probability that this walker reaches $y=x$ at time $t$ without going to the origin $y=0 ; p_{2}(t)$ is the probability that this walker 
first reaches the origin at time $t ; p_{3}(x, t)$ is the probability that this walker first passes at the origin at time $t$ without passing through $y=x ; p_{4}(x, t)$ is the probability that this walker with an absorbing boundary at $y=x$ does not pass through the origin up to time $t$ and $p_{5}(t)$ is the probability that this walker does not reach the origin up to time $t$. Eq. (30) is cumbersome in form but simple in nature: The formula for $P_{2 n}(x, t)$ is just a finitetime generalization of Eq. (27), namely it corresponds to the situation when a walker has performed $n$ oscillations around the link $(x-1, x)$, and at time $t$ a walker, or his remains, is to the left of $x$. Eq. (31) has been constructed similarly and describes the situation with a walker to the right of $x$ at time $t$. The convolution structure of Eqs. (30) and (31) suggests to apply the Laplace transform. Indeed, $\tilde{P}_{n}(x, s)=\int_{0}^{\infty} d t e^{-s t} P_{n}(x, t)$, satisfy

$$
\tilde{P}_{2 n}(x, s)=\tilde{p}_{1}(x, s)\left(\tilde{p}_{2}(s)\right)^{n}\left(\tilde{p}_{3}(x, s)\right)^{n-1} \tilde{p}_{4}(x, s)
$$

and

$$
\tilde{P}_{2 n+1}(x, s)=\tilde{p}_{1}(x, s)\left(\tilde{p}_{2}(s)\right)^{n}\left(\tilde{p}_{3}(x, s)\right)^{n} \tilde{p}_{5}(s) .
$$

Fortunately, the probabilities $\tilde{p}_{j}$ have been already computed [21]:

$$
\begin{aligned}
\tilde{p}_{1}(x, s) & =\frac{\operatorname{sh}(\sqrt{s})}{\operatorname{sh}(x \sqrt{s})} \\
\tilde{p}_{2}(s) & =e^{-\sqrt{s}} \\
\tilde{p}_{3}(x, s) & =\frac{\operatorname{sh}((x-1) \sqrt{s})}{\operatorname{sh}(x \sqrt{s})} \\
\tilde{p}_{4}(x, s) & =\frac{1-\tilde{p}_{3}(x, s)}{s} \\
\tilde{p}_{5}(s) & =\frac{1-\tilde{p}_{2}(s)}{s} .
\end{aligned}
$$

It is in principle possible now to compute various $P_{n}(t)$. For example, the contribution to $P_{1}(t)$ from links with $k \geq 2$ is

$$
\begin{aligned}
\tilde{P}_{1}(s)-\tilde{P}_{1}(1, s) & =\frac{1-e^{-\sqrt{s}}}{s} \operatorname{sh}(\sqrt{s}) \int_{2}^{\infty} \frac{d x}{\operatorname{sh}(x \sqrt{s})} \\
& =\frac{1-e^{-\sqrt{s}}}{s^{3 / 2}} \operatorname{sh}(\sqrt{s}) \ln (\operatorname{cth}(\sqrt{s}))
\end{aligned}
$$




$$
\simeq \frac{\ln (1 / s)}{2 \sqrt{s}} \quad(s \rightarrow 0)
$$

where the contribution from the first link $(0,1)$ is $P_{1}(1, t)=1-1 / \sqrt{\pi t}$, which gives the asymptotic value of

$$
P_{1}(t) \simeq 1-1 / \sqrt{\pi t}+\frac{\ln t}{\sqrt{4 \pi t}} \quad(t \rightarrow \infty)
$$

We now turn to determination of the scaling functions. In the long-time limit, $(t \rightarrow \infty)$, corresponding to $(s \rightarrow 0)$, Eq. (32) becomes

$$
\tilde{P}_{2 n}(x, s) \simeq \frac{e^{-n \sqrt{s}}}{s} \frac{1}{x^{2}}\left(1-\frac{1}{x}\right)^{n-1},
$$

which then implies

$$
\tilde{P}_{2 n}(s)=\int_{2}^{\infty} d x \tilde{P}_{2 n}(x, s) \simeq \frac{e^{-n \sqrt{s}}}{n s} .
$$

Performing the inverse Laplace transform [22], one finds

$$
P_{2 n}(t)=\frac{1}{n} \operatorname{Erfc}\left(\frac{n}{\sqrt{4 t}}\right) .
$$

Indeed the anticipated scaling behavior suggested earlier is confirmed with the scaling function

$$
\Phi_{\text {even }}(z)=z^{-1} \operatorname{Erfc}(z / 2)
$$

In particular, the limiting forms are

$$
P_{2 n}(t) \simeq \begin{cases}\frac{1}{n}-\frac{1}{\sqrt{\pi t}}, & n \ll \sqrt{t}, \\ \frac{1}{n^{2}} \sqrt{\frac{4 t}{\pi}} \exp \left(-\frac{n^{2}}{4 t}\right), & n \gg \sqrt{t} .\end{cases}
$$

For the odd distribution, a similar scaling form is expected:

$$
P_{2 n+1}(t)=\frac{1}{\sqrt{t}} \Phi_{\text {odd }}^{\prime}\left(\frac{t}{n^{2}}\right)
$$

When $(s \rightarrow 0)$, we can use the naive expansion as previously but we should keep the upper limit finite, $\leq s^{-1 / 2}$, since the integrand logarithmically diverges on the upper limit: 


$$
\begin{aligned}
\tilde{P}_{2 n+1}(s) & \simeq \frac{e^{-n \sqrt{s}}}{\sqrt{s}} \int_{2}^{s^{-1 / 2}} \frac{d x}{x}\left(1-\frac{1}{x}\right)^{n} \\
& \simeq \frac{e^{-n \sqrt{s}}}{\sqrt{s}} E_{1}(n \sqrt{s}),
\end{aligned}
$$

with the exponential integral $E_{1}(y)=\int_{y}^{\infty} d u u^{-1} \exp (-u)$. Making use of Eq. (43) one gets another relation for $\tilde{P}_{2 n+1}(s)$,

$$
\begin{aligned}
\tilde{P}_{2 n+1}(s) & =\int_{0}^{\infty} d t e^{-s t} \frac{1}{\sqrt{t}} \Phi_{\text {odd }}^{\prime}\left(\frac{t}{n^{2}}\right) \\
& =n \int_{0}^{\infty} \frac{d T}{\sqrt{T}} e^{-q T} \Phi_{\text {odd }}^{\prime}(T),
\end{aligned}
$$

with $q=n^{2} s$.

Thus we obtain the Laplace transform of the function $\Phi_{\text {odd }}^{\prime}(T) / \sqrt{T}$,

$$
\int_{0}^{\infty} \frac{d T}{\sqrt{T}} e^{-q T} \Phi_{\text {odd }}^{\prime}(T)=\frac{e^{-\sqrt{q}}}{\sqrt{q}} \int_{\sqrt{q}}^{\infty} \frac{d y}{y} e^{-y} .
$$

Performing the inverse Laplace transform, we get

$$
\frac{\Phi_{\text {odd }}^{\prime}(T)}{\sqrt{T}}=\int_{0}^{T} \frac{d \tau}{2 \tau} \operatorname{Erfc}\left(\frac{1}{\sqrt{4 \tau}}\right) \frac{1}{\sqrt{\pi(T-\tau)}} \exp \left(-\frac{1}{4(T-\tau)}\right) .
$$

Performing asymptotic analysis yields

$$
P_{2 n+1}(t) \simeq \begin{cases}\frac{\ln \left(t / n^{2}\right)}{\sqrt{4 \pi t}}, & n \ll \sqrt{t}, \\ \frac{1}{n^{2}} \sqrt{\frac{2 t}{\pi}} \exp \left(-\frac{n^{2}}{t}\right), & n \gg \sqrt{t} .\end{cases}
$$

Notice that in the both limiting cases, $P_{2 n+1}(t) \ll P_{2 n}(t)$.

It proves insightful to compute the moments of even and odd distributions, $M_{\text {even }}^{p}(t)=$ $\sum_{n \geq 1}(2 n)^{p} P_{2 n}(t)$ and $M_{\text {odd }}^{p}(t)=\sum_{n \geq 0}(2 n+1)^{p} P_{2 n+1}(t)$. Asymptotically, it is easy to compute even moments

$$
M_{\mathrm{even}}^{p}(t)=E_{p} t^{p / 2}, \quad E_{p}=\frac{4^{p} \Gamma\left(\frac{p+1}{2}\right)}{p \sqrt{\pi}} .
$$

Eq. (49) is valid only for $p>0$ (when $(p \rightarrow+0)$, the prefactor $E_{p}$ diverges). To determine the most interesting zero moment, i.e. the total number of links crossed even times $M_{\text {even }}^{0}(t)=$ $\sum_{1}^{\infty} P_{2 n}(t)$, we use the the Laplace transform of Eq. (39) to obtain $M_{\text {even }}^{0}(s) \simeq \ln (1-$ $\exp (-\sqrt{s})) / s \simeq \ln (1 / s) / 2 s$ and eventually, 


$$
M_{\text {even }}^{0}(t) \sim(\gamma+\ln t) / 2
$$

with $\gamma \cong 0.577215$ the Euler constant. This result is consistent with a direct summation of $P_{2 n}(\infty)=n^{-1}$ up to $n=\sqrt{t}$. For negative $p$, even moments are finite, $M_{\text {even }}^{p}(\infty)=2^{p} \zeta(1-p)$.

Odd moments behave similarly, $M_{\text {odd }}^{p}(t)=O_{p} t^{p / 2}$. A lengthy computation gives the prefactor

$$
O_{p}=\frac{2^{2 p+1} \Gamma\left(1+\frac{p}{2}\right)}{\pi} \int_{0}^{1} d \mu\left(1-\mu^{2}\right)^{\frac{p-1}{2}} \int_{0}^{\mu} d \theta \frac{\theta^{p+1}}{1-\theta^{2}}
$$

Eq. (51) is valid for all nonnegative $p$, and in particular the (average) total number of links crossed odd times approaches a surprising constant

$$
M_{\mathrm{even}}^{0}=-\frac{1}{2 \pi} \int_{0}^{1} d \mu \frac{\ln \left(1-\mu^{2}\right)}{\sqrt{\left(1-\mu^{2}\right)}}=\ln 2 .
$$

Thus although the odd part of the $P_{n}$ distribution approaches zero as $t \rightarrow \infty$, the moments remain nontrivial.

\section{Simulation Results}

To test the above predictions we performed numerical simulations of the voter model with different initial concentrations, in one dimension. The rich behavior predicted by the meanfield and the exact results was confirmed by the simulation results. We studied the fraction of persistent voters for the case $c_{+}=1 / 3$, and we found the decay exponents $\beta_{+}=0.54$, and $\beta_{-}=0.23$ for the minority and the majority opinion, respectively. These values are in excellent agreement with Eq. (24).

We also confirmed that each of the four functions $P_{2 n}^{ \pm}(t)$ and $P_{2 n+1}^{ \pm}$can be rewritten in a scaling with the scaling variable $n / \sqrt{t}$. The dominance of the even part of the distribution $P_{2 n}>P_{2 n+1}$, is nicely demonstrated by Fig. (4) (one realization of a system of $10^{6}$ sites) and the asymptotics of the even scaling function Eq. (25) are verified.

We performed also simulations for the extreme case $c_{+} \rightarrow 0$, where one site with initial opinion + is in a sea of - opinions. As shown above, this problem is equivalent to the 
average number of times a link is crossed by two annihilating random walkers. We show on Fig. (4) the even and odd scaling functions for $10^{8}$ realizations of this system. The asymptotic results Eqs.(42) found in the simplified problem of one random walker in the presence of an absorbing boundary conditions are verified up to numerical prefactors. In particular, the even scaling functions of Fig. (4) is found to behave asymptotically $(z \rightarrow 0)$ as $\Phi_{\text {even }}(z) \simeq 5 /(4 z)$ to be compared with $\Phi_{\text {even }}(z) \simeq 1 / z$ of Eq. (41).

\section{SUMMARY}

We have investigated the voter model, one of the simplest models of non-equilibrium statistical mechanics with non-conserved dynamics. We have introduced the set of quantities $P_{n}(t)$, defined as the fraction of voters who changed their opinion $n$ times up to time $t$. The distribution $P_{n}(t)$ was shown to exhibit a scaling behavior that strongly depends on the dimension of the system and on the opinion concentrations. For $d>2$, the system does not coarsen, and the distribution is Poissonian. In one-dimension, We have solved for $P_{n}(t)$ in the extreme case when the minority opinion is infinitesimal. The case when the minority phase occupies a negligible volume has been studied in the classical work [23] for the conserved dynamics and has proven very important in the development of the theory of phase ordering kinetics [1]. It would be very interesting to generalize the extreme-case solution to arbitrary $d$.

The quantity $P_{n}(t)$ reflects the history of the coarsening process. Knowledge of this distribution enables insight into interesting quantities such as the fraction of consistent or "frozen" sites, the fraction of sites with their original opinion, and the average number of changes in a site. This study suggests that $P_{n}(t)$ is a tool for investigations of coarsening processes in more realistic models. It possible that a Poissonian $P_{n}(t)$ generally describes systems that do not coarsen, while asymmetric distributions which are pronounced near the origin correspond to coarsening systems. 


\section{ACKNOWLEDGEMENTS}

It is a pleasure to thank S. Redner for fruitful discussions. E. B. was supported in part by NSF under Award Number 92-08527 and by the MRSEC Program of the National Science Foundation under Award Number DMR-9400379. L. F. was supported by the Swiss National Science Foundation.

\section{FIGURE CAPTIONS}

Fig. 1 Scaling for the symmetric case in one-dimension. The quantity $t^{1 / 2} P_{n}(t)$ is plotted versus $n / t^{1 / 2}$ for different times, $t=10^{3}, 10^{4}, 10^{5}$.

Fig. 2 The distribution function $P_{n}(t)$ versus $n$ at time $t=2000$ in 2D.

Fig. 3 The fraction of persistent voters in $2 \mathrm{D}, P_{0}(t)$ versus $\ln ^{2} t$. An average over 300 samples of linear size $L=10^{3}$ for $c_{+}=c_{-}=1 / 2$ (solid line) and over 50 samples of linear size $L=10^{3}$ for $c_{+}=1-c_{-}=1 / 4$ (dashed line).

Fig. 4 The even and odd distribution functions for different $c_{+}$in $1 \mathrm{D} \cdot t^{1 / 2} P_{n}(t) /\left(c_{+} c_{-}\right)$is plotted versus $n / t^{1 / 2}$. Different scaling functions correspond to the even (upper curves) and the odd (lower curves) parts of the distribution. The solid lines correspond to the case $c_{+}=1 / 4$ for one sample of linear size $L=10^{6}$. The dashed lines correspond to the 


\section{REFERENCES}

[1] A comprehensive recent review of the coarsening dynamics is given by A. J. Bray, Adv. Phys. 43, 357 (1994).

[2] D. S. Fisher and D. A. Huse, Phys. Rev. B 38, 373 (1988).

[3] R. J. Glauber, J. Math. Phys. 4, 294 (1963).

[4] A. Coniglio and M. Zanetti, Europhys. Lett. 10, 575 (1989).

[5] H. K. Janssen, B. Schaub, and B. Schmittmann, Z. Phys. B 73, 539 (1989).

[6] A. J. Bray and B. Derrida, Phys. Rev. E 51, 1633 (1995).

[7] A. J. Bray, Phys. Rev. B 41, 6724 (1990).

[8] D. A. Huse, Phys. Rev. B 40, 304 (1989).

[9] P. L. Krapivsky, Phys. Rev. A 45, 1067 (1992).

[10] S. N. Majumdar and D. A. Huse, Phys. Rev. E 52, 270 (1995).

[11] B. Derrida, A. J. Bray, and C. Godreche, J. Phys. A 27, L357 (1994).

[12] P. L. Krapivsky, E. Ben-Naim, and S. Redner, Phys. Rev. E 50, 2474 (1994).

[13] D. Stauffer, J. Phys. A 27, 5029 (1994).

[14] J. L. Cardy, J. Phys. A 28, L19 (1995); E. Ben-Naim, cond-mat 9412048; M. Howard, cond-mat 9510053.

[15] B. Derrida, J. Phys. A 28, 1481 (1995); B. Derrida, V. Hakim, and V. Pasquier, Phys. Rev. Lett. 75, 751 (1995).

[16] A. J. Bray, B. Derrida, and C. Godreche, Europhys. Lett. 27, 175 (1994).

[17] T. M. Liggett, Interacting Particle Systems (Springer, New York, 1985).

[18] L. Frachebourg and P. L. Krapivsky, cond-mat 9508123. 
[19] B. U. Felderhof, Rep. Math. Phys. 1, 215 (1970) and 2, 1 (1971); D. Bedeaux, K. E. Shuler, and I. Oppenheim, J. Stat. Phys. 2, 1 (1970).

[20] R. J. Rubin and G. H. Weiss, J. Math. Phys. 23, 250 (1982).

[21] W. Feller, An Introduction to Probability Theory, Vols. 1 and 2 (Wiley, New York, 1971).

[22] M. Abramowitz and I. Stegun, Handbook of Mathematical functions, (Dover, New York, 1972).

[23] O. M. Todes, J. Phys. Chem. (Soviet) 20, 629 (1946); I. M. Lifshitz, Zh. Eksp. Teor. Fis. 42, 1354 (1962) [JETP 15, 939 (1962)]; I. M. Lifshitz and V. V. Slyozov, J. Phys. Chem. Solids 19, 35 (1961); C. Wagner, Z. Elektrochem. 65, 581 (1961). 


\section{FIGURES}

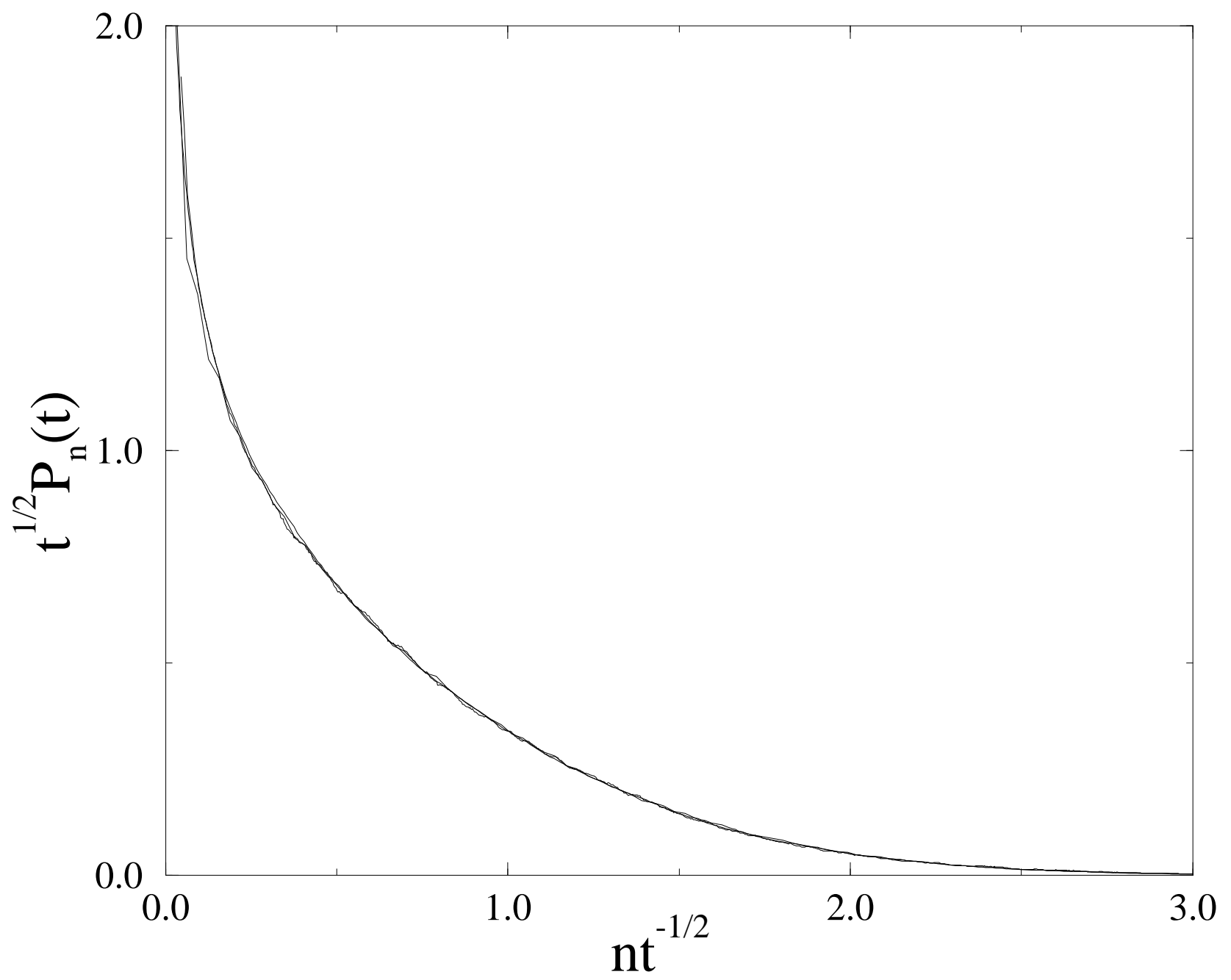

Figure 1 


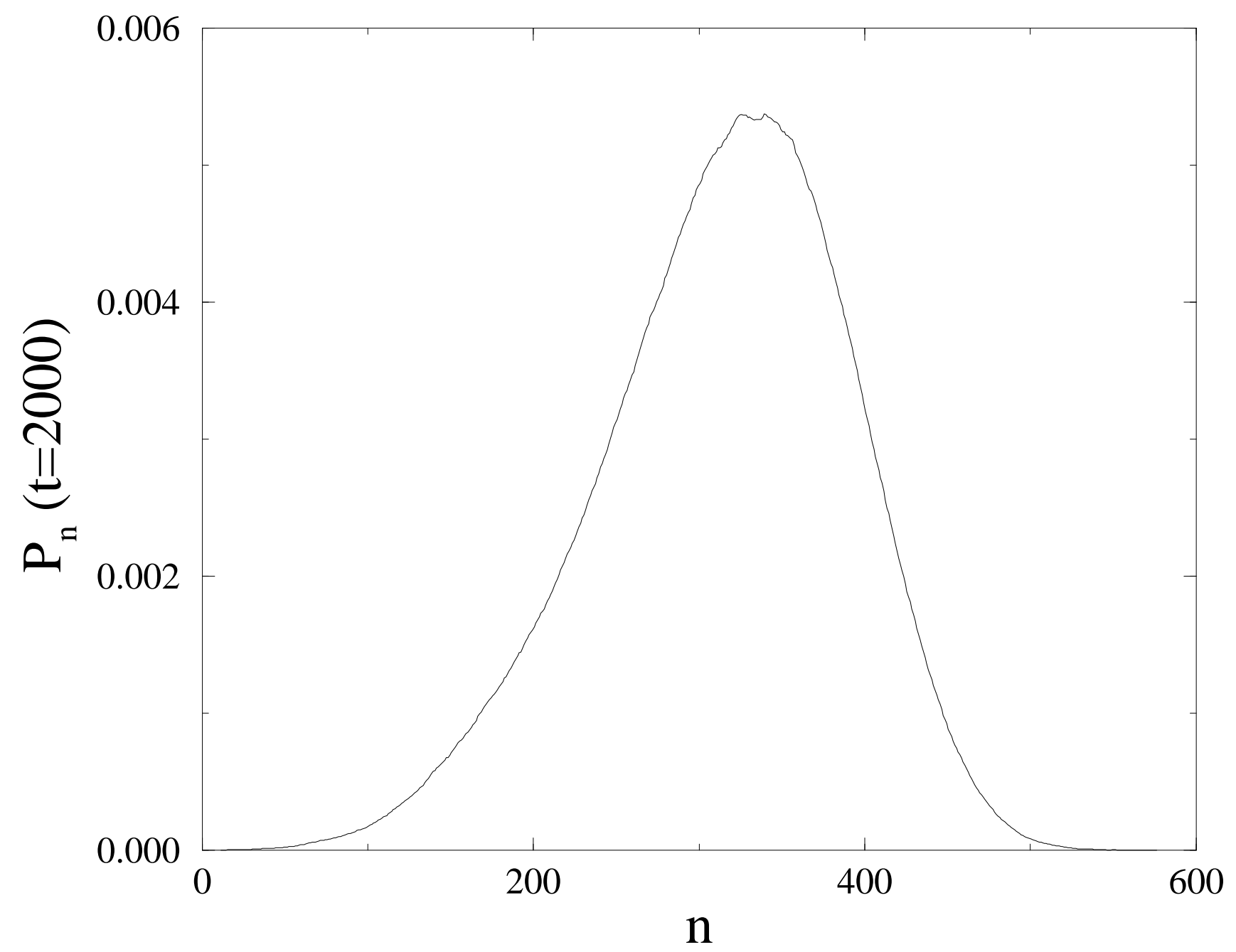

Figure 2 


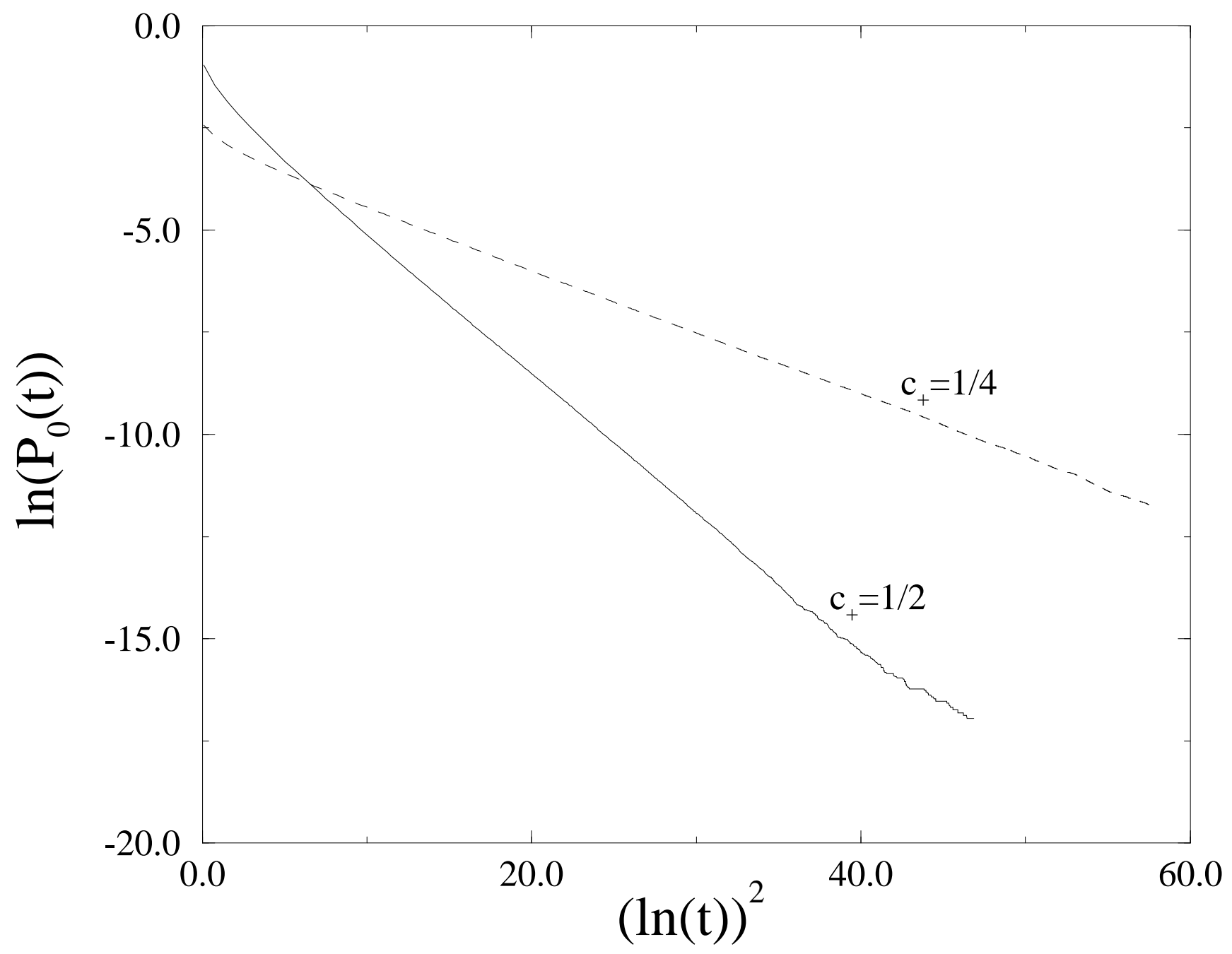

Figure 3 


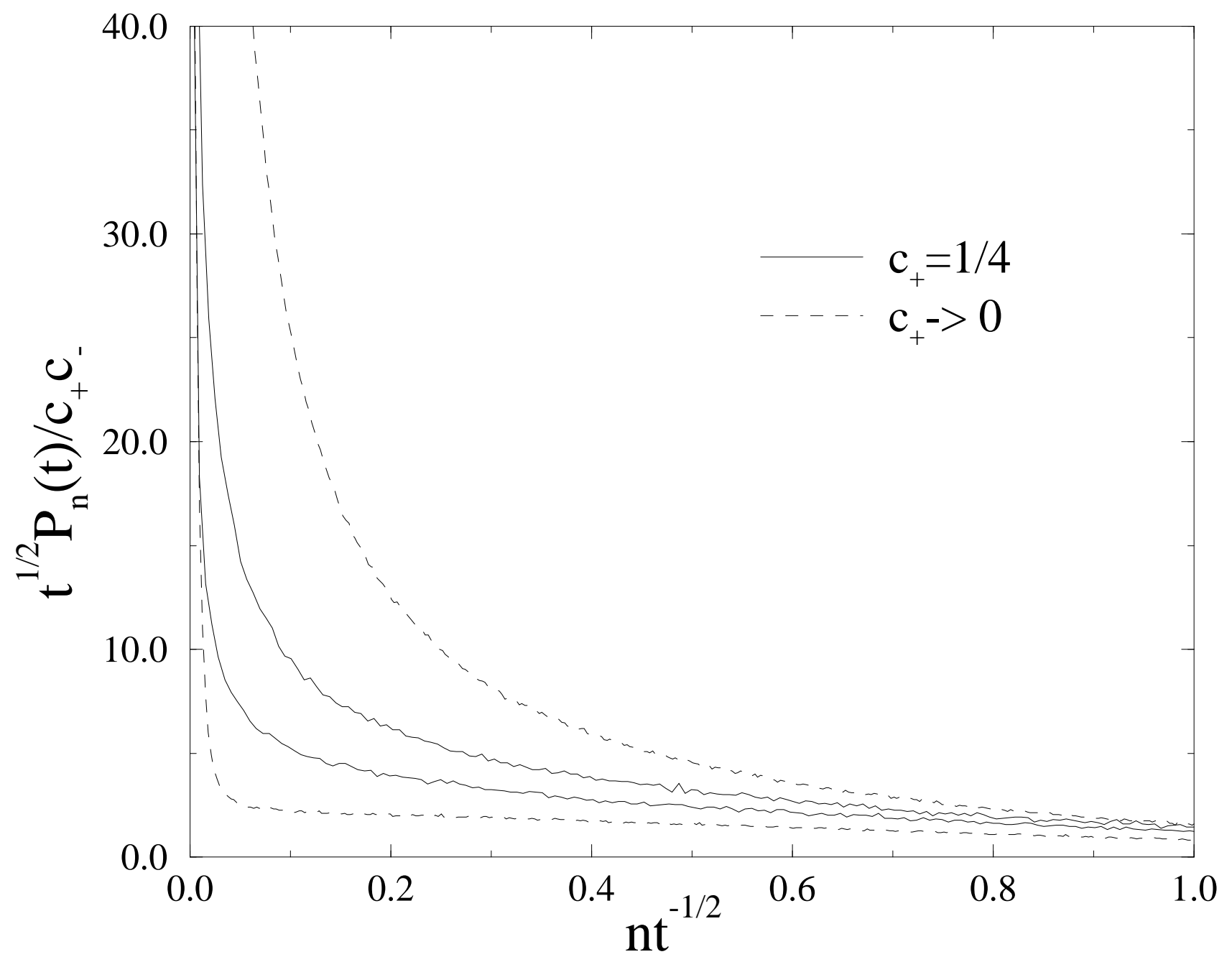

Figure 4 